DOI: $10.21767 / 2471-9838.100018$

\section{Nanoparticles Restrictions in Environmental Cleanup}

\section{Abstract}

Environmental contamination with a variety and mixed pollutants are a problematic issue worldwide. If the contaminated sites are left without any satisfactory remediation, it leads to the successive threaten of ecosystems, then human life. So, applications of nanotechnology in environmental remediation are encouraged because of the novel properties (e.g. special structure, very large surface area and greater reactivity), whereas, "Nano" may be more than just "small". Also, environmental nanotechnology restraint is due to lack of full understanding characterization, fate and transport of such ultrafine materials in the environment. The objective of this work is to point major challenges facing environmental nanoscience and urges developing eco-friendly techniques to ensure good quality of life.

Keywords: Ecosystems; Nano; Remediation; Environment; Contamination

\section{Moustafa Gamal Snousy ${ }^{1}$ and Zawrah $\mathrm{MF}^{2}$}

\author{
1 Petrotread Co., Anwar Al Moftty St. 1, \\ Abbas El Akkad, Nasr City, Cairo, Egypt \\ 2 National Research Centre, Center of \\ Excellence, Nano Group, Dokki, Cairo, \\ Egypt
}

Corresponding author:

Moustafa Gamal Snousy

E moustafa_gamal93@yahoo.com

Petrotread Co., Anwar Al Moftty St. 1, Abbas El Akkad, Nasr City, Cairo, Egypt.

Tel: +201006517228

Citation: Snousy MG, Zawrah MF. Nanoparticles Restrictions in Environmental Cleanup. Nano Res Appl. 2017, 3:1.

\section{Introduction}

Due to a widespread of industrial activities, an extraordinary number of contaminants are released into the environment. That can pollute soil, air, and water, as well as cause deforestation, biodiversity losses, soil degradation and harm to human health [1]. For example, when pollutants are released into aquatic ecosystems, direct (toxic) effects on aquatic biota are possible, as well as, indirect effects in ecological communities by complex relationships (i.e. through entering food chain). Many of these pollutants are known or suspected as carcinogens, mutagens and may alter ecosystem function [2]. Therefore, a variety of environmental treatment techniques have been designed, using bioremediation, phytoremediation, physical and chemical remediation and so on. The recognition that "traditional" methods of contaminated treatment (e.g. disposal to landfill, isolation, pump-and-treat) are not sustainable (or indeed effective) in many situations, that lead to a massive increase in research into the development of alternative treatment technologies for environmental remediation [3]. The development of effective strategies for the prevention and remediation of contamination is of key importance that can help to preserve and restore the integrity of natural habitats [4].

Nanoscience and nanoengineering can provide cost-effective options for removal of myriads of harmful agents presented in the environment [5]. Besides the treatment of chemical contaminants, nanotechnologies can play a significant role in developing emerging technologies for detection and decontamination of harmful biological agents, since nanoparticles (NPs) provide a very good flexibility for both treatment methods.

\section{Environmental cleanup: Benefits and limits of nano-based technologies}

Nanoscale particles proved the potential of novel nanomaterials for treatment of surface waters, groundwater, wastewater, soil and sediments contaminated by heavy metals, microorganisms, organic and inorganic solutes. In addition, they can cost effectively address some difficult problems connected to soil and groundwater decontamination [6]. The high surface-area-to-mass ratios (i.e. specific surface area) of these materials can benefit any technologies that rely on reactions at solid-water and solid-gas interfaces. Such technologies include adsorption used for water and exhaust-gas treatment as well as photocatalytic processes for contaminant degradation. Nanoscale sizes can also influence the chemical reactivity of materials by the predominance of nearsurface regions with compositions distinct from bulk regions and increasing the contribution of interfacial free energy to the free energy of dissolution-precipitation reactions [7]. 
Nano-scale zero-valent particles (NZVPs) are considered widely used in environmental remediation. The presence of these nanoparticles in the environment may include nanoscale colloids with special properties that clearly affect in the fate, transport, transformation and bioavailability of pollutants in various ecosystems. The understanding of its risk to human or ecological health is still limited. So, the impact assessment of the nanomaterials on the environment and human health has attracted worldwide attention [8]. Although NZVPs have been successfully used in the past few years in treating wide classes of environmental contaminants including toxic metals, organic and inorganic compounds [9], the following challenges are still existing.

NZVPs have demonstrated and exhibited high reactivity in remediating many hazardous compounds such as non-aqueous phase liquids and heavy metals. Reducing the particle size of granular materials from $(\mathrm{mm})$ to $(\mathrm{nm})$, increases surface area and thus chemical reactivity. The nanoparticles small size allows both easy to be transported in the subsurface via injection or direct push in slurry form to contaminated zones under pressure or gravity in the view of treatment $[6,10]$. Delivering the NZVPs to the contaminant zone is essential for the success of remediation. But, its transport properties are poor due to Brownian motion, the density of particles, long range magnetic attractive forces and ionic strength, which increase the aggregation of NZVPs and result in filtration of NZVPs in subsurface [11].

NZVPs recirculation in subsurface by using injection/extraction wells or by pneumatic fracturing, that leads to an increase in ambient $\mathrm{pH}$ and decrease in solution redox potential usually occurs, yielding an alkaline and reducing environment. In addition, clogging of porous media around the injection points/wells (also ionic strength-dependent), decreases in hydraulic conductivity, groundwater flow changes, as well as alterations to microbial distributions. In addition to changes to the ambient environment from NZVPs injection, the NZVPs also undergo chemical and/or biological transformation. For instance, increased $\mathrm{pH}$ conditions favor the formation of hydroxides (sorption, agglomeration and mobility of the nanoparticles) will be strongly dictated by $\mathrm{pH}$ [12], based on the stability complex constants, which can also affect porosity conditions [13]. It was found that the injection of NZVPs caused a significant change total solid (TS) and suspended solid (SS) concentrations in groundwater. In a groundwater environment, the dissolved oxygen concentration is usually very low. Therefore; the predominant electron receptor is water. It is worthy of mention that the groundwater possesses a high ionic strength in the testing site that may cause the agglomeration of NZVPs and limit its movement [8].

NZVPs are usually supposed to be highly mobile in porous medium because they are ultra-smaller than the relevant pore spaces, but this is an oversimplification. In general, the mobility of nanoparticles in saturated porous media is determined by the product of the nanoparticle collisions number with the porous medium per unit transport distance and the probability that any collision will result in removal of the nanoparticle from the flow system (i.e. the sticking coefficient). Collisions may result from three processes: Brownian diffusion, interception and gravitational sedimentation. For nanoparticles, under conditions that are typical of environmental porous media, Brownian diffusion is the dominant collision process. For particles, greater than $\sim 400 \mathrm{~nm}$ and with high densities, gravity effects can become important [14].

NZVPs transport in subsurface is extremely limited due to agglomeration that making it difficult to distribute into the contaminated zone during remediation [15]. Nanoparticles aggregate rapidly (in a few minutes) in water, resulting in micro-, millimeter-scale or larger aggregates. The aggregated particles are not typically transportable through the contaminated zone matrix and thus are not deliverable to the targeted polluted zones. In addition, aggregation will result in a decrease in the NZVPs specific surface area which, in turn, would affect its reactivity. Both particle-particle interaction (agglomeration) and particlecollector interaction (deposition) affect their transport in porous media $[16,17]$. That subsequently led to loss in reactivity and decreased environmental mobility. These processes are thought to be concentration-dependent. Concentrations used are sitedependent due to variations between source zone construction, contaminant plume extents, contaminant type, hydrogeological conditions, etc. and may occur through several mechanisms such as Brownian diffusion, interception, and gravitational sedimentation [18]. NZVPs must be stable to aggregation and have a very low deposition (or filtration) rate, as otherwise their mobility in the subsurface will be greatly limited [19].

Nanoparticles quantitative information detailed relating to transport behavior in porous media remains lacking yet [20]. Contaminated medium composition and environmental conditions in natural systems such as the presence of humic acid (the mobility is increased in the presence of humic acid) in subsurface, as well as ionic strength of the resident water, influences and modifies nanoparticle mobility. Increasing the ionic strength enhances the deposition of the nanoparticles, in other words, the mobility of NZVPs is decreased with ion concentrations increase [21]. The addition of salt enhances the deposition of the nanoparticles [22], and increases NZVPs aggregation [13]. Under conditions of higher ionic strength, attractive van der Waals forces are dominant over repulsive electrostatic interactions, leading to enhanced aggregation and thus reduced mobility of the nanoparticles. For most cases, the mobility of the nanoparticles is relatively low, lower flow rates again led to reduced mobility [22].

Because of its ionic strength, sea water modifies the surface charge of nanomaterials generating more particle collisions, aggregation, and precipitation [23]. On the other hand, the ecological impact and movement of NPs on whole terrestrial ecosystems remains unreported. Whereas, soil and sediments solution chemistry (e.g. ionic strength, $\mathrm{pH}$, and presence of natural organic matters) strongly affects the interactions between NPs and the solid media, thus influencing the balance between the free migration of particles and the deposition of NPs.

Bare NZVPs may be essentially non-mobile in porous media due to particle aggregation and attachment [24]. Also, bare 
NZVPs may not be readily dispersible in both aqueous or organic solvents because of intrinsic magnetic interactions and their high surface energies [9]. So, modification of particles with dispersants is required to improve transport in contaminated zone, but any such modification is generally believed to decrease the reactivity of nanoparticles [25].

NZVPs unstable characteristic has been a disadvantage in practical application, despite the expectation of an enhanced reactivity. Since NZVPs is only stable in a reducing environment [13], nanosized particles react spontaneously with atmospheric oxygen [26]. It has been ever increasing benefits to maintain the NZVPs stability in air without significant reactivity sacrifice. However, such remediative methods limited for shallow groundwater plumes in unconsolidated aquifers, precluding their use for a vast range of contaminated sites worldwide [11]. However, the high surface energy makes NPs reactive and easy to get oxidized in the open air, which results in decreasing or completely losing their functions and properties [9]. This demonstrated encapsulating NZVPs in a hydrophobic coating to secure from other water constituents. This protected the core of nanoparticles from oxidation in air (pure NZVPs is pyrophoric and burns spontaneously in air [26] and enhanced their dispersion stability in organic solvents [9].

NZVPs surface (in direct reduction) is still not certain whether plays any important role in the process of contaminant removal in NZVPs $/ \mathrm{H}_{2} \mathrm{O}$ systems. In other words, although NZVPs are currently regarded as an established remediation technology, its efficiency is still to be demonstrated [10]. Also, the produced byproducts during the reduction processes can be even more toxic than their parent compounds. There have also been some concerns regarding NZVPs potential for environmental persistency, both in terms of the original NZVPs 'parent' particles or applied coatings as well as any formed, transformed, or 'daughter' particles, particularly combined with its potential for subsurface transport. So, the hazard assessment of NPs quantitative nanoecotoxicological data is required [13].

The reduction rate tends to decrease with increased elapse time due to the formation of oxide layers (aerobic corrosion of NZVPs results in the formation of an oxide layer on the particle surface which reduces particle reactivity [24], that can block the NZVPs surface active sites. So, the reductive rate of contaminants may be low, thus a long hydraulic retention time is needed for complete reduction of the target contaminates [27]. However, NZVPs has been extensively used for the decontamination of groundwater, its efficacy in soil remediation is being increasingly considered [24]. The reactivity in soils is complicated as recalcitrant organic chemicals are sorbed to soil minerals and organic matter [15].

The nanoparticles appeared to be mobile in the subsurface, but mobility decreased relative over time, with very little mobility after 13 hours. Because of this time-dependent mobility, high advective groundwater velocities would be needed to deliver the nanoparticles substantial distances from the point of injection. Advective delivery of the reactive nanoparticles did not appear to treat a considerable portion of the contaminations present within the permeable portion of the aquifer zones because the injected fluid simply away pushed the contaminated water. The implications extend to all sites where the intent is to treat dissolved mass by injecting a reactive substrate into a series of wells, if the abundance of immediately accessible stationary mass relative to mobile (dissolved) mass is low, as is often the case for dissolved plumes in fractured rock settings and granular aquifers [28].

Nanoparticles with high chemical reactivity and low solubility will likely have limited transport in general [13], and NZVPs which are highly sorbed to soil and sediment surfaces are not expected to partition into the water column as dissolved solutes. Also, other processes may play additional roles in NZVPs behavior and migration which are not yet well understood, including unforeseen exposure pathways. Thus, long-term environmental behavior and migration are still virtually unknown. Generally, nanoparticles interact with microorganisms present in soil and groundwater through passive and active mechanisms that alter the chemical form and hence the groundwater transport and soil retention characteristics of the nanoparticles. This will ultimately affect the human exposure route and toxicity. During biological treatment, some nanoparticles have been found to inhibit or even prevent biological activity. A reduction of biological activity by toxic nanoparticles could decrease the contaminant removal effectiveness of the entire facility failing effluent discharge limits [12]. It is likely that enhanced bioremediation was involved at the application of NZVPs testing site because of its strongly reducing conditions [8].

Because of the potential environmental risks, NZVPs use may not necessarily be the 'best' solution in field scale applications. Depending on the contaminated site and ambient environmental conditions, such as for example sites where groundwater flow is directed to surface water bodies or sensitive aquatic ecosystems (e.g. wetlands), especially under high hydraulic flow conditions, or in other cases where decision makers actively include a 'minimal level of uncertainty' as a choice criteria when selecting a remediation option. In these cases, alternatives assessment may (or may not) reveal other treatment options with 'safer' and potentially less 'risky' options [13].

In addition, alternatives assessment may also be a potential decision making tool for NZVPs in which, different treatment options may be reviewed and the option with the 'lowest' level of potential risk or degree of uncertainty may be chosen as the 'best available technology' for a given site or remediation objectives. In this way, the risk evaluators or decision makers can clearly state the choice or value criteria for the decision in hand, after which they may then choose the 'best' or 'safest' option based on available data. The important processes and pathways of NPs in the environment are shown in Figure 1. This illustrates the unintentional and intentional release of NPs into the environment. Whereas, NZVPs are directly injected into polluted sites with organics and heavy metals, humans can be either directly influenced by NPs through exposure to air, soil or water, or indirectly by consuming plants or animals which have accumulated NP [29]. Ontological approaches towards studying and understanding the interaction of nanoparticles 


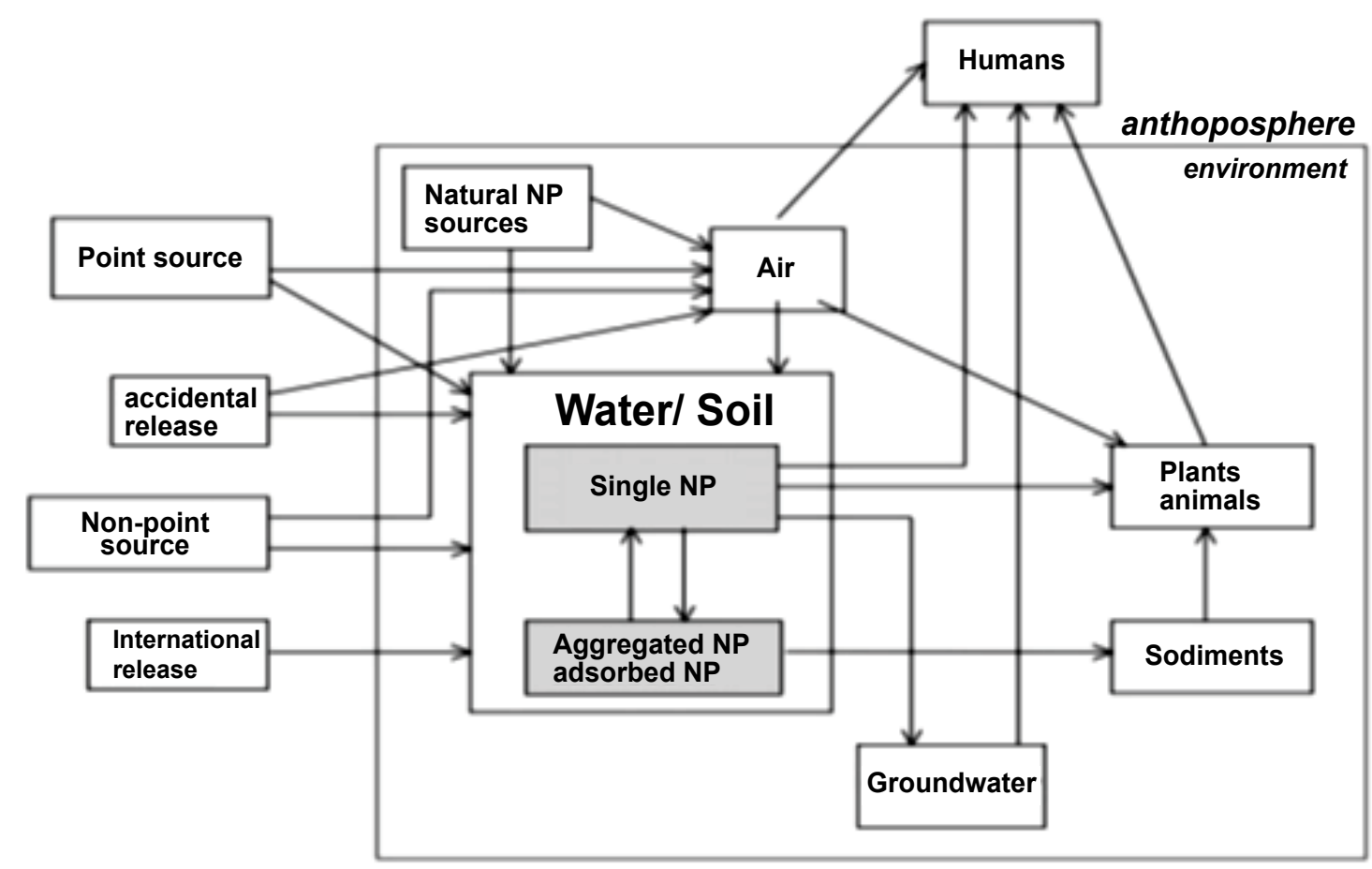

Figure 1 Nanoparticle pathways into the environment, reactions in the environment and exposure of humans [31].

with the human body, nanoparticles dynamics in air and aquatic systems, composition-dependent disposition, and dispersion of nanoparticles, short- and long-term effects of nanomaterials with the human body, immunotoxicity and phototoxicity of nanoscale materials will address some of the societal issues of nanotechnology [30].

Nevertheless, when trying to balance the potential benefits and risks of NZVPs as an environmental remediation strategy for a given site, it may indeed be the 'best' solution in some cases. This may be for instance some sites which may require very fast remediation, as in the case of highly toxic contaminants, or other sites which require treatment in especially hard-to-reach places which are not suited for other techniques [31].

From all the above clarifications, research needs development of eco-friendly 'smart colloids' or 'Nano-colloidal materials' which are based on green chemistry in environmental cleanup. This significance arises from the statement that "as colloidal fines can carry contaminants adsorbed on their surface, they can also carry beneficial molecules to a contaminated site".

\section{Conclusion}

During the few decades, the occurrence of environmental threat has resulted in extensive research, introduced nanotechnology in the environmental cleanup, but it is still in the first stage. High reactivity of NZVPs alone is not sufficient to ensure effective remediation. To date, there is still uncertainty regarding the technology's potential for full-scale field application. The use of nanomaterials in environmental applications requires a better understanding of their mobility, bioavailability and toxicity. Furthermore, no consensus has been reached on many fundamental issues, including NZVPs stability, and longevity under subsurface conditions. This work includes subjects on risk assessment/toxicity, exploring the ecological and ecotoxicological assessments, characterization and stability, toxicity, fate, transport and limitations facing NZVPs in the ecosystems. Future pollution remediation nanomaterials-based techniques may include pollution transport by nanoparticles which will have medical, radiological and even national defense implications in terms of human health, safety, and the environment. 


\section{References}

1 Brito de Figueiredo MC, Rodrigues GS, Caldeira-Pires A, Morsyleide de FR, Antônio SAF, et al. (2010) Environmental performance evaluation of agro-industrial innovations e Part 2: Methodological approach for performing vulnerability analysis of watersheds. J Clean Prod 18: 1376-1385.

2 Fleeger JW, Carman KR, Nisbet RM (2003) Indirect effects of contaminants in aquatic ecosystems: A review. Sci Total Environ 317: 207-233.

3 Cundy AB, Hopkinson L, Whitby RLD (2008) Use of iron-based technologies in contaminated land and groundwater remediation: $\mathrm{A}$ review. Sci Total Environ 400: 42-51.

4 Antizar LB, Galil NI (2010) Biofilm and colloidal biomass dynamics in a shallow sandy contaminated aquifer under in-situ remediation conditions. Int Biodeterior Biodegradation 64: 331-338.

5 Bystrzejewska PG, Golimowski J, Urban PL (2009) Nanoparticles: Their potential toxicity, waste and environmental management. Waste Manag 29: 2587-2595.

6 Caliman FA, Robu BM, Smaranda C, Pavel VL, Gavrilescu M (2010) Soil and groundwater cleanup: Benefits and limits of emerging technologies: A review. Clean Techn Environ Policy 13: 241-268.

7 Kanatzidis MG, Poeppelmeier KR (2007) Report from the third workshop on future directions of solid-state chemistry: The status of solid-state chemistry and its impact in the physical sciences. Progr Solid State Chem 36: 1-133.

8 Wei YT, Wu SC, Chou CM, Che CH, Tsai SM, et al. (2010) Influence of nanoscale zero-valent iron on geochemical properties of groundwater and vinyl chloride degradation: A field case study. Water res 44: 131- 140.

9 Wang G, Sun Q, Zhang Y, Fan J, Ma L (2010) Sorption and regeneration of magnetic exfoliated graphite as a new sorbent for oil pollution. Desalination 263: 183-188.

10 Noubactep C, Care S (2010) On nanoscale metallic iron for groundwater remediation. Short communication. J Hazardous Mater 182: 923-927.

11 Scott TB, Popescub IC, Cranea RA, Noubactep C (2011) Nano-scale metallic iron for the treatment of solutions containing multiple inorganic contaminants. J Hazard Mater 186: 280-287.

12 Brar SK, Verma M, Tyagi R D, Surampalli RY (2010) Engineered nanoparticles in wastewater and wastewater sludge-Evidence and impacts. Waste Manag 30: 504-520.

13 Grieger KD, Fjordbøge A, Hartmann NB, Eriksson E, Bjerg PL, et al. (2010) Environmental benefits and risks of zero-valent iron nanoparticles ( $\mathrm{nZVI}$ ) for in situ remediation: Risk mitigation or tradeoff? J Contam Hydrol 118: 165-183.

14 Logan BE (1999) Environmental transport processes. John Wiley \& Sons, New York, NY.

15 Noubactep C, Licha T, Scott TB, Fall M, Sauter M (2009) Exploring the influence of operational parameters on the reactivity of elemental iron materials. J Hazard Mater 172: 943-951.
16 Phenrat T, Kim HJ, Fagerlund F, Illangasekare T, Lowry GV (2010) Empirical correlations to estimate agglomerate size and deposition during injection of a polyelectrolyte-modified $\mathrm{Fe}^{\circ}$ nanoparticle at high particle concentration in saturated sand. J Contam Hydrol 118: 152-164.

17 Zhao W, Wang QQ, Liu F, Qiu GH, Tan WF, et al. (2010) Pb Pb $^{2+}$ adsorption on birnessite affected by $\mathrm{Zn}^{2+}$ and $\mathrm{Mn}^{2+}$ pretreatments. J Soils Sediments 10: 870-878.

18 Tratnyek PG, Johnson RL (2006) Nanotechnologies for environmental cleanup. Nano Today 1: 44-48.

19 Tiraferri A, Chen KL, Sethi R, Elimelech M (2008) Reduced aggregation and sedimentation of zero-valent iron nanoparticles in the presence of guar gum. J Colloid and Interface Sci 324: 71-79.

20 He F, Zhang M, Qian T, Zhao D (2009) Transport of carboxymethyl cellulose stabilized iron nanoparticles in porous media: Column experiments and modeling. J Colloid Interface Sci 334: 96-102.

21 Lin YH, Tseng HH, Wey MY, Lin MD (2010) Characteristics of two types of stabilized nano zero-valent iron and transport in porous media. Sci Total Environ 408: 2260-2267.

22 Ben MT, Dror I, Berkowitz B (2010) Transport of metal oxide nanoparticles in saturated porous media. Chemosphere 81: 387-393.

23 Peralta Videa JR, Zhao L, Lopez Moreno ML, de la Rosa G, Hong J, et al. (2011) Nanomaterials and the environment: A review for the biennium 2008-2010 a review. J Hazard Mater 186: 1-15.

24 Cullen LG, Tilston EL, Mitchell GR, Collins CD, Shaw LJ (2011) Assessing the impact of nano and micro-scale zerovalent iron particles on soil microbial activities: Particle reactivity interferes with assay conditions and interpretation of genuine microbial effects. Chemosphere 82: 1675-1682.

25 Darko-Kagya K, Khodadoust AP, Reddy KR (2010) Reactivity of lactatemodified nanoscale iron particles with 2,4- dinitrotoluene in soils. $J$ Hazard Mater 182: 177-183.

26 Tiehm A, Silke K, Yuri K, Aharon G (2009) Chloroethene dehalogenation with ultrasonically produced air-stable Nano iron. Ultrason Sonochem 16: 617-621.

27 Feng J, Lim TT (2005) Pathways and kinetics of carbon tetrachloride and chloroform reductions by Nano-scale $\mathrm{Fe}$ and $\mathrm{Fe} / \mathrm{Ni}$ particles: Comparison with commercial micro-scale Fe and $\mathrm{Zn}$. Chemosphere 59: 1267-1277.

28 Bennett P, He F, Zhao D, Aiken B, Feldman L (2010) In situ testing of metallic iron nanoparticle mobility and reactivity in a shallow granular aquifer. J Contam Hydrol 116: 35-46.

29 Lyons K, Whelan J (2010) Community Engagement to Facilitate, Legitimize and Accelerate the Advancement of Nanotechnologies in Australia. Nanoethics 4: 53-66.

30 Vaseashta A, Vaclavikova M, Vaseashta S, Gallios G, Roy P, et al. (2007) Nanostructures in environmental pollution detection, monitoring, and remediation. Sci Technol Adv Mater 8: 47-59.

31 Nowack B, Bucheli TD (2007) Occurrence, behavior and effects of nanoparticles in the environment: A review. Environ Pollut 150: 5-22. 